Int. J. Dev. Biol. 50: 17-26 (2006)

doi: $10.1387 / \mathrm{ijdb} .052080 \mathrm{fm}$

Original Article

\title{
Interplay between FGF10 and Notch signalling is required for the self-renewal of pancreatic progenitors
}

\author{
FRANCISCO MIRALLES*, LUCIANE LAMOTTE, DOMINIQUE COUTON and RAJIV L. JOSHI \\ Institut Cochin, Department of Genetics, Development and Molecular Pathology, INSERM U567, CNRS UMR8104, Université René \\ Descartes Paris V, France
}

\begin{abstract}
Recent studies have shown that persistent expression of FGF10 in the developing pancreas of transgenic mice results in enhanced and prolonged proliferation of pancreatic progenitors, pancreatic hyperplasia and impaired pancreatic differentiation. These studies have also suggested that FGF10 prevents the differentiation of pancreatic progenitors by maintaining persistent Notch signalling. Here, we provide experimental evidence sustaining the capacity of FGF10 to induce the proliferation of pancreatic precursors, while preventing their differentiation. Using explant cultures of E10.5 isolated dorsal pancreatic epithelium, we found that FGF10 maintained Notch activation and induced the expansion of pancreatic precursors while blocking their differentiation. In addition, by using a $\gamma$-secretase inhibitor, we were able to down-regulate the expression of Hes 1, a target gene of the Notch pathway in explant cultures of pancreatic epithelium treated with FGF10. In such explants, the effect of FGF10 on the proliferation and maintenance of pancreatic progenitors was suppressed. These results demonstrate that activation of the Notch pathway is required as a downstream mediator of FGF10 signalling in pancreatic precursor cells.
\end{abstract}

KEY WORDS: pancreas development, FGF10, Notch pathway, proliferation, differentiation

\section{Introduction}

The mammalian pancreas is a mixed organ composed of exocrine and endocrine glands. In mice the pancreas develops from two evaginations of the primitive gut endoderm giving rise to the dorsal and ventral pancreatic anlagen. The first sign of pancreas morphogenesis is the formation of the dorsal pancreatic anlagen at embryonic day 9.5 (E9.5), followed shortly thereafter by the formation of the ventral anlagen (E10). The pancreatic precursor cells present in these anlagen are characterized by the co-expression of the transcription factors Pdx1, Nkx2.2, Nkx6.1 and $\mathrm{p} 48 / \mathrm{Ptf} 1 \alpha$. Between E9.5-13.5, these cells undergo a phase of intense proliferation leading to an expanded precursors pool which subsequently and sequentially differentiate, giving rise to all the endocrine and exocrine cells of the mature pancreas (Edlund, 2002; Jensen, 2004).

The Notch pathway has been identified as the mechanism controlling the self-renewal of the pancreatic precursors by preventing premature differentiation. In this pathway the transmembrane ligands Delta or Jagged expressed in adjacent cells (or in the same cell) activate the Notch receptors. Ligand activation leads to the cleavage of the intracellular domain of Notch (IC), which interacts with the RBPjk protein. This complex transactivates target genes including the Hairy Enhancer of Split (HES) family of bHLH repressors (Beatus and Lendahl, 1998). The HES repressors generally act by down-regulating pro-differentiation factors such as Achaete and Scute in Drosophilaproneurons (Fisher and Caudy, 1998), MyoD in mammalian myoblasts (Kuroda et al., 1999) and Neurogenin 3 (Ngn3) in a neuroendocrine context (Lee et al., 2001). The studies of the genetic inactivation of different components of this pathway, Hes1 (Jensen et al., 2000), Ngn3 (Gradwohl et al., 2000) and RBPjk (Apelqvist et al., 1999) as well as mice over-expressing $\mathrm{Ngn} 3$ in early pancreatic progenitors (Apelqvist et al., 1999), collectively show that Notch signalling controls the choice between differentiated endocrine cell and progenitor cell fates. According to this model the inactivation of the Notch receptor or signalling results in Ngn3 activation, leading to premature endocrine cell differentiation at the expense of pancreatic progenitors expansion and exocrine cell differentiation. In contrast, cells with active Notch signalling remain as undifferentiated progenitor cells that would proliferate, undergo morphogenesis and late differentiation. Recent studies have

Abbreviations used in this paper: DBA, Dolihcos Biflorus Agglutinin lectin; e, embryonic day; FGF, fibroblast growth factor; HES, hairy enhancer of split; ngn, neurogenin; PP, pancreatic polypeptide.

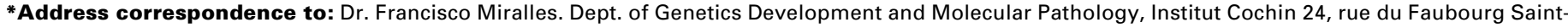
Jacques, 75014 Paris, France. Fax: +33-1-4441-2421. e-mail: miralles@cochin.inserm.fr
} 
shown that Notch activation prevents not only endocrine differentiation but also acinar cell differentiation (Hald etal., 2003;Murtaugh et al., 2003;Esni et al., 2004).

Although the key role of the Notch pathway in the maintenance of the self-renewal capacity of the pancreatic precursors seems well established, the coordination between this pathway and the signals controlling the growth, morphogenesis and cytodifferentiation remains poorly understood. The earlier studies on pancreas development have revealed the importance of epithelialmensenchymal interactions in the control of pancreas development. These studies have shown that in the absence of mesenchyme the isolated pancreatic epithelium fails to grow and differentiates only into endocrine cells. In the absence of mesenchyme, morphogenesis is also abolished (Golosow and Grobstein, 1962; Wessels and Cohen, 1967). More recent studies have shown that mesenchyme can regulate the ratio between endocrine and exocrine cell differentiation (Rutter et al., 1978;Gittes et

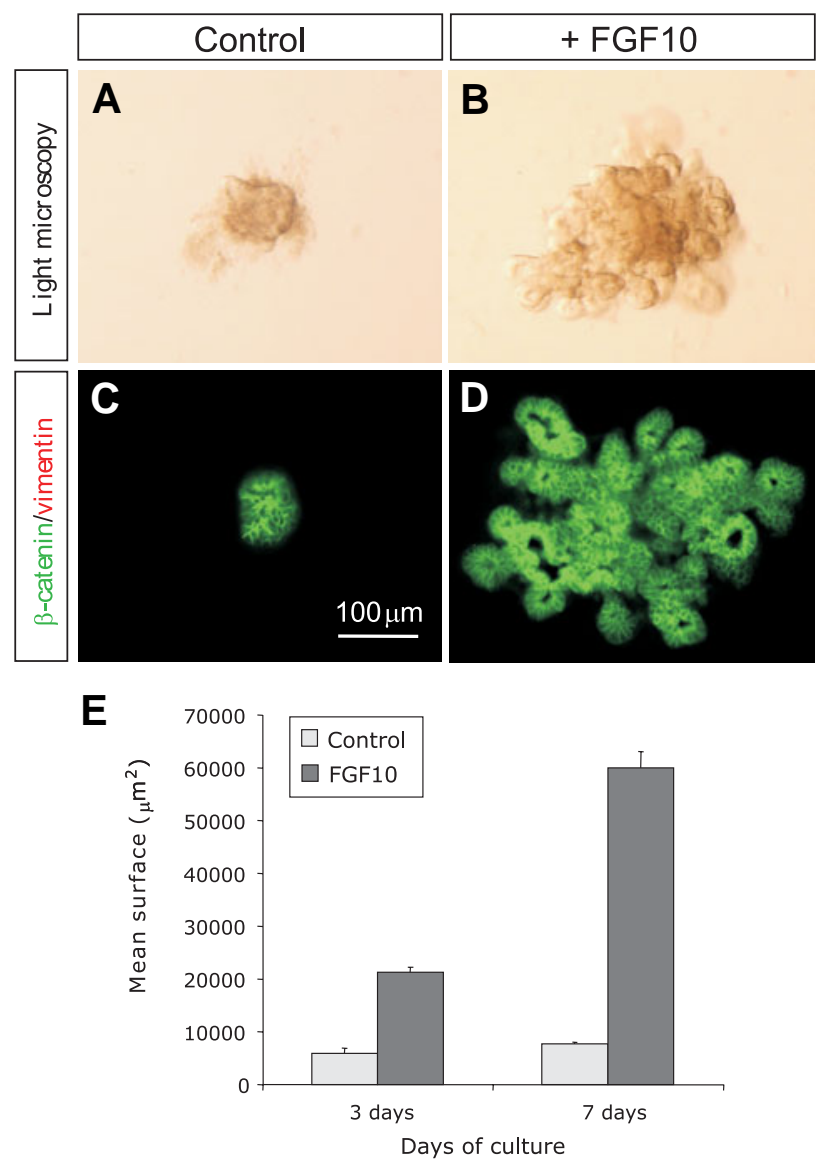

Fig. 1. Effects of FGF10 on the growth and morphogenesis of isolated E10.5 dorsal pancreatic epithelia. (A,B) Isolated pancreatic epithelia grown in vitro for 7 days, without and with $100 \mathrm{ng} / \mathrm{ml} \mathrm{FGF10.} \mathrm{(A)} \mathrm{The}$ untreated epithelium fails to grow and remains as a dense agregate of cells with occasional budding. (B) After 7 days of culture, the FGF10-treated epithelium shows an irregular structure formed by buds, cysts and tubules. (C,D) $\beta$-catenin/vimentin staining of the pancreatic epithelium after 7 days of culture. (E) Quantitative analysis of the size of the isolated pancreatic epithelia after 3 and 7 days of culture. The bars represent the mean \pm SEM of 6 epithelia. al., 1996;Miralles et al., 1998).

The mesenchyme produces and secretes a large variety of growth factors which act as mediators of epithelial-mesenchymal interactions in many organs. Some of them, i.e. EGF, HGF, TGF $\beta$ and different FGFs, have been implicated in pancreatic growth and differentiation (Kim and MacDonald, 2002). Among these, the FGFs signalling through the receptor FGFR2b seems to play a critical role in pancreatic organogenesis. Indeed, mice expressing a dominant negative form of FGFR2b and mutant null-mice for FGF1R2b or for its ligand FGF10, show severe pancreatic hypoplasia (Celli etal., 1998; Revest etal., 2001;Bhushan etal., 2001).

We had shown in a previous study that different FGFs (FGF1, FGF7 and FGF10) signalling through the FGFR2b, induced the proliferation of the isolated rat embryonic pancreatic anlagen. Moreover after 7 days of culture with these FGFs most of the cells of the pancreatic epithelia appeared to have differentiated into acinar cells (Miralles et al., 1999). However, the pancreatic epithelia treated with FGF10 retained some cells which did not stain for any markers of endocrine or exocrine pancreatic differentiation. Although we could not characterize these cells at that time, we presumed they were undifferentiated progenitors.

Interestingly, two recent studies have shown that persistent expression of the FGFR2b high-affinity ligand FGF10 in the developing pancreas of transgenic mice resulted in enhanced and prolonged proliferation of pancreatic progenitors, pancreatic hyperplasia and impaired pancreatic cell differentiation. Both studies provide evidence that FGF10 could maintain the activation of the Notch pathway throughout the developing pancreatic epithelium, thereby preventing the differentiation of the pancreatic progenitors (Norgaard et al., 2003; Hart et al., 2003).

These new observations prompted us two characterize more thoroughly the effects of FGF10 on the development of isolated mouse embryonic pancreatic epithelia in vitro. Moreover, we have used the in vitro cultures to examine the effects of FGF10 on the maintenance of the Notch pathway in an active state in the pancreatic precursor cells. Our data show that in the isolated embryonic pancreatic epithelium, FGF10 induces the proliferation of the pancreatic progenitors and blocks their differentiation. We provide also experimental evidence demonstrating that the effect of FGF10 on the self-renewal of the pancreatic progenitors is dependent on active Notch-signalling.

\section{Results}

\section{Growth of pancreatic rudiments in growth factor reduced Matrigel}

Dorsal pancreatic rudiments from mouse embryos were taken at $E 10.5$, depleted of their surrounding mesenchyme and cultured for 3 or 7 days in growth factor reduced Matrigel. The pancreatic rudiments were grown either in the absence or presence of FGF10.

Pancreatic epithelia grown without FGF10 did not significantly increase in size over the culture period (Fig. $1 \mathrm{~A}$ and E). Occasionally small buds were observed on the surface of the rudiments. After seven days of culture the pancreatic rudiments were doubleimmunostained with anti- $\beta$-catenin and anti-vimentin antibodies (Fig. 1C). The rudiments were negative for vimentin indicating that no contaminant mesenchymal cells were left after the depletion procedure. The $\beta$-catenin staining showed that these rudi- 


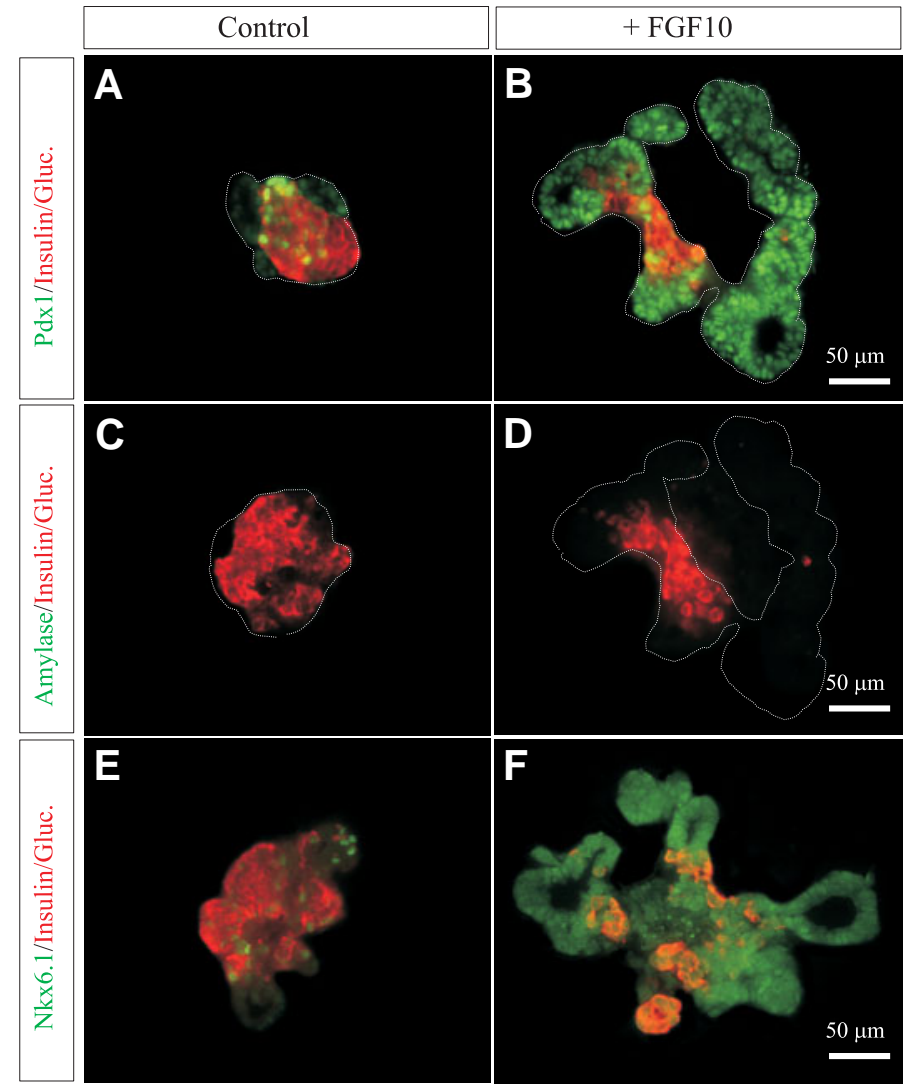

Fig. 2. Expansion of the pancreatic precursors in the FGF10-treated pancreatic epithelia. (A,B) Double immunofluorescence staining for Pdx1 (green) and insulin+glucagon (red). (A) In the untreated epithelia, most cells are insulin or glucagon positive and only a few show Pdx1 staining. (B) The majority of the cells in the FGF10-treated epithelium stain for Pdx 1 and only a few for insulin or glucagon. (C,D) Double immunofluorescence staining for amylase (green) and insulin+glucagon (red). Both untreated and FGF10-treated epithelia are negative for amylase staining (the contours of the explants have been outlined with white lines). (E) In the untreated epithelium, only a few cells are positive for Nkx6.1 staining (green) and most of these are also positive for insulin or glucagon staining. (F) In the FGF10-treated epithelia, the majority of the cells are positive for Nkx6.1 staining and only a few co-stain with insulin or glucagon.
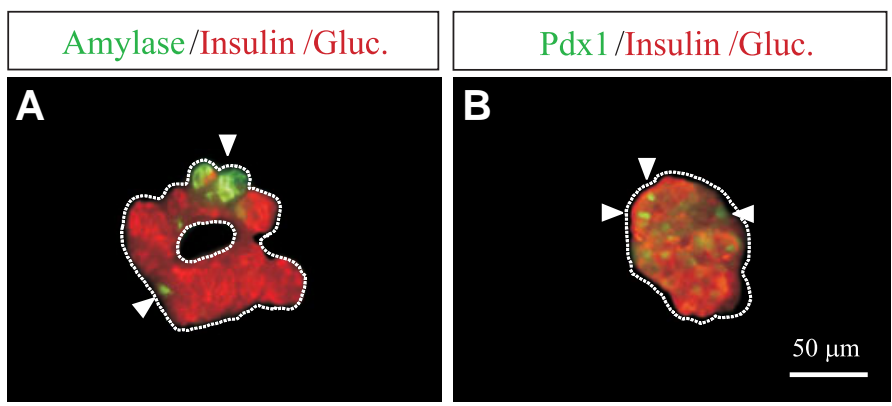

Fig. 3. Development of isolated pancreatic epithelia after 7 days of culture. (A) Double-immunofluorescence staining for amylase (green) and insulin+glucagon (red). After 7 days of culture the isolated pancreatic epithelium differentiates into a majority of endocrine cells and a few acinar cells. Ductal cell were not detected. (B) Double immunofluoresce for Pdx 1 (green) and insulin+glucagon (red). ments were formed by tightly associated epithelial cells surrounding a small central lumen. The buds protruding from the rudiment were also formed by tightly clustered cells surrounding in some cases a small lumen.

The pancreatic rudiments treated with FGF10 grew rapidly and after 7 days of culture they had underwent a 10 -fold increase in size (Fig. $1 \mathrm{~B}$ and $\mathrm{E}$ ). By the second day of culture the FGF10treated explants started to show morphological changes. Multiple buds appeared in the surface of the rudiments which progressively transformed into tubular structures that invaded the surrounding gel (Fig. 1B). The $\beta$-catenin immunostaing showed that these tubular structures were formed by single layered epithelial cells surrounding a lumen (Fig. 1D). Vimentin staining was negative, indicating that there was no contamination by mesenchymal cells and also, that FGF10 did not have any trophic effect on mesenchyme cells that could eventually remain after the depletion procedure.

\section{Analysis of pancreatic differentiation after three days of culture}

After three days of culture the pancreatic epithelial rudiments grown in the absence of FGF10 stained positively for glucagon and insulin (Fig. 2A). We did not detect any cells staining for somatostatin or pancreatic polypeptide (PP). Staining for amylase (Fig. 2C) and carboxypeptidase A (not shown) was negative and we also could not detect any cells staining positively for DBA (Dolihcos Biflorus Agglutinin) lectin (specific for ducts in the pancreas). Pdx1 staining was detected in some cells (Fig. 2A). A few cells showing strong Pdx1 staining, co-stained positively for insulin, indicating that they were $\beta$-cells. Cells showing low levels of Pdx1 staining, never co-stained with other pancreatic markers, suggesting that they were undifferentiated cells. Similar results were obtained with Nkx6.1 staining (Fig. 2E). The quantitative analysis showed that after three days of culture, the cells expressing glucagon and insulin represented respectively $60 \%$ and $30 \%$ of the relative cell surface in the rudiments (Fig. 5A). The undifferentiated cells represented only $10 \%$ of the rudiments surface.

Pancreatic epithelia grown in the presence of FGF10 for three days stained positively for glucagon and insulin (Fig. 2B) and were negative for amylase (Fig. 2D), somatostatin, PP, carboxypeptidase $A$ and DBA lectin (not shown). In these rudiments most cells showed strong staining for Pdx1 (Fig. 2B) and Nkx6.1 (Fig. 2F). Only a small fraction of the Pdx1/Nkx6.1 staining cells, co-stained also for insulin, indicating that the majority of them were undifferentiated cells. The quantitative analysis indicated that after three days of culture in the presence of FGF10, glucagon and insulin-expressing cells represent only $15 \%$ and $5 \%$ respectively of the total cell surface in the rudiments. Thus, the majority of the cells remained undifferentiated (Fig. 5A).

\section{Analysis of pancreatic differentiation after seven days of culture}

After seven days of culture the untreated pancreatic epithelial rudiments had not significantly increased in size with respect to the initiation of the culture. In these rudiments $85 \%$ of the total cell surface stained positively for glucagon+insulin. Amylase staining cells composed the remaining 15\% (Fig. 3A and Fig. 5B). Pdx1 staining (Fig. 3B) and Nkx6.1 staining (not shown) were found only in a few cells. Double immunofluorescence stainings ( $\mathrm{Pdx} 1 /$ 
insulin or Nkx6.1/insulin) showed that in these rudiments the Pdx1 and Nkx6.1 positive cells always co-stained with insulin (not shown). Thus after seven days of culture the untreated pancreatic epithelia had completely differentiated. These rudiments were composed essentially by endocrine cells (85\%) and a few, acinar cells (15\%). We did not detect any cells staining for DBA lectin in these explants and we also failed to detect the specific ductal products CFTR and Carbonic anhydrase // by RT-PCR (data not shown). Cells staining for somatostatin or the PP were also absent.

The isolated pancreatic epithelia treated with FGF10 showed a 10-fold increase in size after seven days of culture. In these rudiments cells expressing glucagon or insulin (Fig. 4 A,B, C) represented respectively $7 \%$ and $3 \%$ of the rudiments surface (Fig. 5B). Amylase expressing cells represented $40 \%$ of the
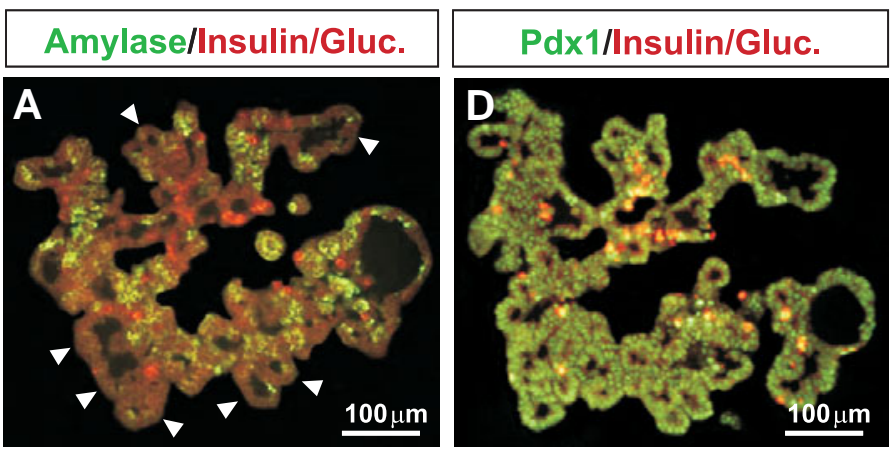

Amylase/lnsulin/Gluc.
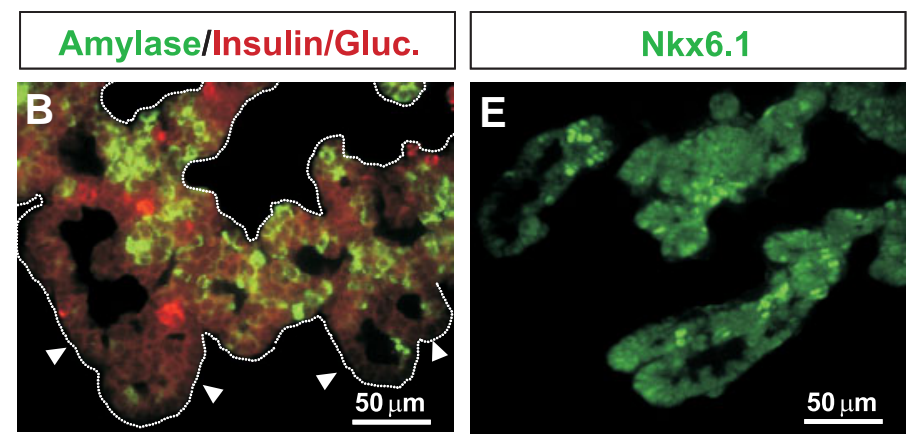

Amylase/Insulin/Gluc.
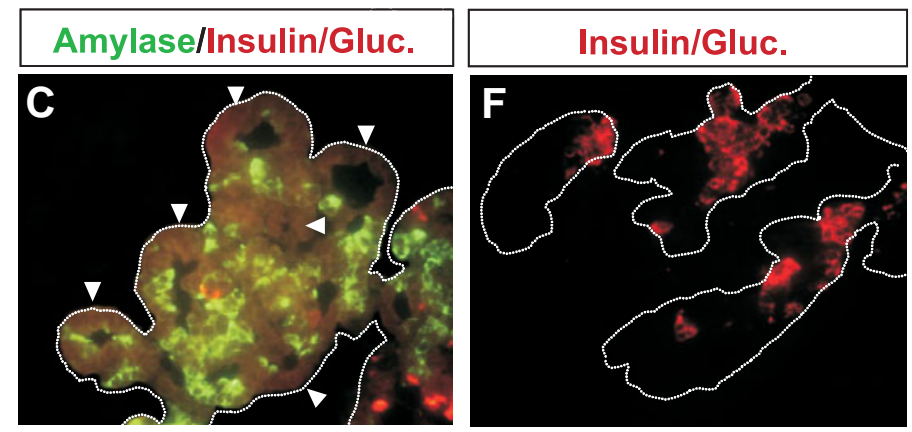

Fig. 4. Development of the isolated pancreatic epithelia after 7 days of culture in the presence of FGF10. (A,B,C) Double-immunofluorescence staining for amylase (green) and insulin+glucagon (red). Althougth many cells appear to have differentiated into acinar cells, there is also a considerable number of cells which do not stain for exocrine or endocrine markers (arrow heads). (D) Immunostaining for Pdx1 (green) and insulin+glucagon (red). (E,F) Same section stained for Nkx6.1 (green) and insulin+glucagon (red). Most cells in the FGF10-treated epithelia are positive for Pdx1 and Nkx6.1. rudiment (Fig. $4 \mathrm{~A}, \mathrm{~B}, \mathrm{C}$ and $5 \mathrm{~B}$ ) and the remaining $50 \%$ was occupied by undifferentiated cells (Fig. 4 A,B,C and 5B). In the explants treated with FGF10 most cells were positive for Pdx1 and Nkx6.1 staining (Fig. $4 \mathrm{D}$ and E). Only a small fraction of these cells stained for insulin or glucagon (Fig. $4 \mathrm{D}$ and F). These results show that after 7 days of culture the pancreatic epithelia treated with FGF10 still have a considerable number of undifferentiated cells. These cells are presumably pancreatic progenitors. As for the untreated epithelia we could not detect cells expressing somatostatin, PP, or ductal markers.

\section{FGF10 stimulates the proliferation of E10.5 isolated pancre- atic epithelium}

To determine the effects of FGF10 on the proliferative capacity of the pancreatic explants, we added BrdU (5-Bromo-deoxyUridine) to the culture medium 3 hours before stopping the cultures. Double immunostainings for $\beta$-catenin and BrdU were performed to calculate the proliferation index in the explants (Fig. $6 \mathrm{C})$. In the untreated pancreatic rudiments after either three or seven days of culture, very few cells were labelled with BrdU (Fig. $6 \mathrm{~A})$. In contrast, pancreatic rudiments treated with FGF10 showed a high BrdU labelling index at both three and seven days of culture (Fig. 6A), indicating that cells were actively proliferating in the FGF10-treated rudiments. Double immunostaining for BrdU and pancreatic cell markers (insulin, glucagon, amylase, Pdx1 and Nkx6.1) allowed us to measure the percent of proliferation for each cell type (Fig. 6B). In the untreated rudiments, cells staining for insulin and glucagon did never co-stain for BrdU and only a few amylase-positive cells were occasionally found to co-stain with BrdU (less than 3\%). In the rudiments treated with FGF10 the endocrine cells were also negative for BrdU staining. On the contrary, cells staining for amylase showed significant BrdU incorporation (15\% of amylase+ cells were BrdU+; Fig. 6B, D and E). Moreover a significant amount of cells (20\% after 7 days of culture) that did not stain for amylase or endocrine hormones, stained for BrdU (Fig. 6 B, D and E). These cells were presumably proliferating precursor cells.

\section{Activation of the Notch pathway in undifferentiated cells of the FGF10-treated rudiments}

The pancreatic rudiments grown in the presence of FGF10 appeared similar to the pancreas of transgenic mice expressing FGF10 under the control of the Pdx1 promoter ( $p P d \times 1-F G F 10$. In these animals pancreatic differentiation was impaired and most cells remained in an undifferentiated state. The expression of Hes 1, a downstream target for Notch, in most of the undifferentiated cells of the transgenic pancreas suggests that this condition could result of persistent activation of the Notch pathway (Hart et al., 2003;Norgaard et al., 2003). Thus, we analyzed by RT-PCR the expression of different components of the Notch pathway in the isolated pancreatic epithelia cultured with or without FGF10. The expression of the Notch1 and Notch 2receptors (Fig. 7A) as well as their membrane bound ligands Jagged 1, Jagged 2 and Delta 1 (not shown) persisted in both the FGF10-treated and untreated pancreatic epithelia trough the culture period. Interestingly, the expression of Hes 1 was rapidly down-regulated in the control epithelia, while it was maintained in the FGF10-treated epithelia (Fig. 7A). In situ hybridization on FGF10-treated rudiments showed that Hes 1 is expressed in a considerable number 
of cells that do not stain for endocrine hormones nor amylase (data not shown).

\section{Inhibition of the Notch pathway blocks FGF10 effects on growth and cell differentiation}

To further demonstrate that the effects of FGF10 on the pancreatic rudiments were mediated by the activation of the Notch pathway, we cultured FGF10 treated rudiments with compound 1, a $\gamma$-secretase inhibitor (Wolfe et al., 1999). The $\gamma$ secretases are intramembrane proteases required for the activation cleavage of Notch receptors following ligand binding (De Strooper et al., 1999). Compound 1, has been previously shown to strongly inhibit Notch activation (De Strooper et al., 1999). Compound 1 was added at 10,25 or $50 \mu \mathrm{M}$ on pancreatic explants that were treated simultaneously with FGF10. RT-PCR analysis demonstrated that compound 1 at $50 \mu \mathrm{M}$, efficiently inhibited Hes 1 expression in FGF10-treated pancreatic epithelia (Fig. 7B). Moreover compound 1 blocked the proliferative effect of FGF10 on the isolated pancreatic epithelium. In some experiments compound 1 was added at the beginning of the culture and in others compound 1 was added after 3 days of culture. In both cases growth arrest was almost immediate. The immunohistochemical analysis showed that in these explants $40 \%$ of the cell surface corresponded to endocrine cells, $50 \%$ to acinar cells and a small proportion (3\%) to undifferentiated cells (Fig. 7C). We could also detect a few cells ( $5 \%$ ) staining positively for the DBA lectin (Fig. 7D). Carbonic anhydrase // transcripts were detected by RT-PCR in these explants. Thus, in these explants a few cells had differentiated into ductal cells.

\section{Lunatic fringe is induced by FGF10 in the isolated pancreatic epithelium}

To further understand how FGF10 can maintain Notch activation in the undifferentiated pancreatic epithelium we analyzed the expression of Lunatic fringe a well known modulator and enhancer of Notch activity. The RT-PCR analysis confirmed that Lunatic fringe is expressed throughout pancreatic development (Fig. 8A). The expression of Lunatic fringe in the developing pancreas appears to be maximal between E12 and E16. Interestingly, this period corresponds to a phase of intense proliferation of the pancreatic progenitors. Moreover, Lunatic fringe expression is downregulated in isolated E10.5 pancreatic epithelium (Fig. 8B), indicating the requirement of a mesenchymal signal for its expression. However, FGF10-treatment restores Lunatic fringe expression (Fig. 8B). Thus, FGF10 could modulate Notch activity by inducing the expression of Lunatic fringe.

\section{Discussion}

To study the effects of FGF10 on the self-renewal of the pancreatic progenitors we have used explant cultures of isolated E10.5 mouse dorsal pancreatic epithelia. The explants were grown into reduced growth factors Matrigel with or without FGF10 and their development analyzed after three or seven days of culture. Our results showed that after three days of culture the untreated pancreatic epithelia did not increase in size while those cultured with FGF10 presented a 4-fold increase in size. The analysis of BrdU incorporation indicated very low proliferation in the untreated rudiments and active proliferation in those receiving
FGF10. The untreated pancreatic epithelia were essentially composed by endocrine cells $(90 \%$ of the total cell surface in the rudiment) and only a few cells remained undifferentiated (10\%). In the FGF10 treated epithelia the reverse was observed: the endocrine cells represented only $15 \%$ of the rudiment surface area while the remaining $85 \%$ was composed of undifferentiated cells. Our results indicate that in the untreated pancreatic epithelia the progenitors cells fail to proliferate and differentiate rapidly. In contrast, in the FGF10-treated epithelia the pancreatic progenitors proliferate actively and remain in an undifferentiated state characterized by the co-expression of the transcription factors Pdx1, Nkx6.1, Ptf1 $\alpha$ and Hes1.

After seven days of culture the untreated pancreatic epithelia did not increase in size and were composed essentially by endocrine cells (85\%) and a few acinar cells (15\%). On the contrary during this period the explants treated with FGF10

A

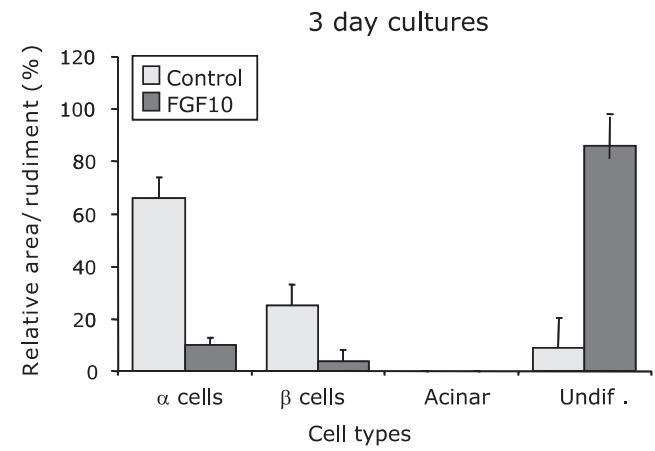

B

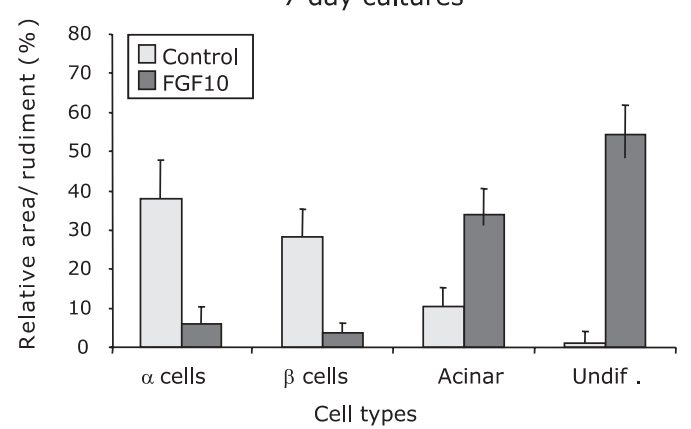

Fig. 5. Quantitative analysis of cell differentiation in the E10.5 isolated pancreatic epithelium grown in vitro with or without FGF10. (A) Analysis of cell differentiation after 3 days of culture. (B) Analysis of cell differentiation after 7 days of culture. The quantitative data are based on single immunostainings for each specific cell marker. Each bar represents the mean \pm SEM of at least 8 epithelia.

underwent a 10-fold increase in size. In these rudiments the endocrine cells represented only $10 \%$ of the total cell surface, most cells being acinar cells (40\%) or undifferentiated cells (50\%). Interestingly BrdU incorporation in these explants concerned always the acinar cells (15\%) and the undifferentiated cells (20\%). Taken together these results indicate that FGF10 has different effects on the proliferation of the pancreatic epithelia. It stimulates the proliferation of pancreatic progenitors and induces the proliferation of differentiated acinar cells. The high BrdU labelling index of the acinar cells in the FGF10 treated rudiments 
suggests that most of these cells arise by proliferation of the few acinar cells which differentiate from the isolated pancreatic epithelia. It is well established that acinar differentiation is dependent on mesenchymal signals (Rutter etal., 1978; Gittes et al., 1996; Miralles et al., 1998). However, in our culture conditions we observed that a few acinar cells form in isolated pancreatic epithelium. The absence of vimentin staining indicated that there was no contamination by mesechyme cells in these cultures. Therefore these cells probably arise from pancreatic progenitors, which were already engaged in acinar differentiation prior to mesenchyme removal. In this respect it is noteworthy that although not detectable by immunohistochemistry, some specific acinar products like carboxypeptidase $A$ can be detected by RT-PCR as early as E9.5 in the pancreatic rudiments of mice (Gittes and Rutter, 1992), (and our own results). An alternative explanation would be that the role of the mesenchyme in pancreatic development is to furnish permissive rather than instructive signals. The FGF10 (as well as other growth factors) secreted by the mesenchyme would allow the expansion of progenitor cells, that subsequently differentiate into exocrine cells.

The trophic effects of FGF10 on the mouse pancreatic epithelia were not unexpected since we had previously shown that FGF10 stimulates the proliferation of the rat embryonic pancreas (Miralles etal., 1999). Moreover FGF10-null mice presented an hypoplastic pancreas (Bhushan et al., 2001), while transgenic mice over expressing FGF10 in the pancreas displayed pancreatic hyperplasia (Hart et al., 2003;Norgaard et al., 2003). It should be mentioned that transgenic expression of a dominant negative FGFR2b under the control of the $P d x 1$ promoter did not lead to a pancreatic phenotype (Hart etal., 2000) and only a week pancreatic hypoplasia was observed in the FGFR2b-null mice (Pulkkinen et al., 2003). These studies seem to contradict the hypothesis that FGF10 could play a major role in the control of the proliferation of the undifferentiated pancreatic epithelia. However it must be noted that FGF10 might signal trough other receptors (Powers et al., 2000). Moreover, other factors like FGF2, FGF7, EGF and HGF are also able to stimulate the proliferation of the embryonic pancreatic epithelia and could compensate the loss of FGF10 signalling (Kim and MacDonald, 2002; Edlund, 2002).

Another important effect of FGF10 is its capacity to maintain a considerable number of cells in an undifferentiated state. As indicated above, after seven days of culture in the presence of FGF10 a considerable number of cells in the isolated pancreatic epithelia did not stain positively for endocrine or exocrine markers of pancreatic differentiation. These cells co-expressed the transcription factors Pdx1, Nkx6.1 and p48/Ptf1 $\alpha$, which is a characteristic of pancreatic progenitors. Moreover, most of the undifferentiated cells in the FGF10-treated epithelia express the transcription factor Hes1. This is another characteristic of pancreatic progenitors and indicates that the Notch pathway is active in these
B

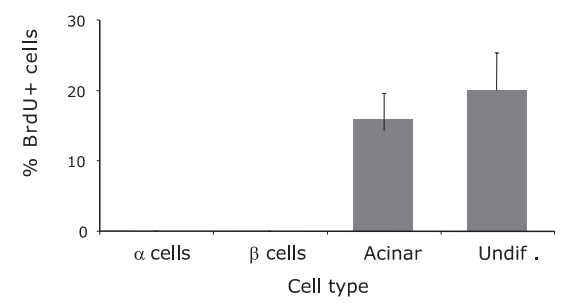

Days of culture

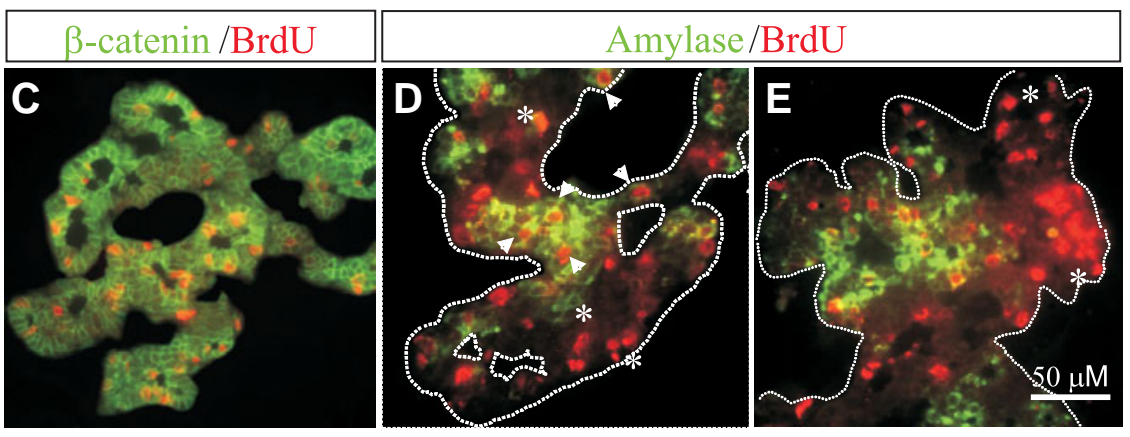

Fig. 6. Cell proliferation analysis. (A) Morphometric analysis of BrdU incorporation in the pancreatic epithelia cultured for 3 or 7 days in the absence or presence of FGF10. Data are BrdU incorporation in the different cell populations of the FGF10-treated pancreatic epithelia. Data are based on specific cell type markers/BrdU double immunostaining. Bars represent the mean \pm SEM of 4 epithelia. (C) Double immunofluorescence staining for $\beta$-catenin/BrdU on a pancretic epithelium cultured for 7 days in the presence of FGF10. (D,E) Amylase/BrdU immunostaining on FGF10-treated pancreatic explants. Amylase+/BrdU+ cells are indicated by arrowheads. Groups of undifferentiated/BrdU+ cells are indicated with asterisks.

cells. These results are similar to what has been reported in the $p P d \times 1$-FGF10transgenic mice, which showed pancreatic hyperplasia, maintenance of pancreatic progenitors in an undifferentiated state and persistent Notch activation (Hart et al., 2003;Norgaard et al., 2003). In our in vitro cultures, acinar differentiation was not so efficiently blocked as it was in the transgenic mice. This could be explained by the fact that in the transgenic mice FGF10 is expressed at the onset of $P d x 1$ expression, that is E8.5. Thus, in these animals, FGF10 is acting on the early pancreatic progenitors. In our study we have used E10.5 pancreatic epithelium. At this stage most cells in the pancreatic rudiment are progenitor cells, but a few cells have differentiated into endocrine cells and others are probably, as mentioned above, already engaged into acinar differentiation. The mitotic effect of FGF10 on the few acinar cells which differentiate spontaneously probably enhances the relative proportion of acinar cells in our model.

The proportion of undifferentiated cells in the mouse explants is increased comparatively to what was previously observed using isolated E11.5 rat dorsal pancreatic epithelium (Miralles et al., 1999). The E11.5 rat and the E10.5 mouse dorsal pancreatic rudiments are very similar in terms of transcription factors expression and cell differentiation status. Thus, the differences are essentially due to different culture conditions. The cultures were done in serum free conditions in previous studies whereas we added $1 \%$ fetal calf serum (FCS) in the present study. We noted 
that despite the presence of FGF10, a minimal amount of other growth factors was necessary to allow the survival and growth of the pancreatic precursors when cultured in the absence of mesenchyme.

Another unexpected observation is the absence of ductal cells in our cultures. Gittes and co-workers have shown that isolated mouse pancreatic epithelium grown in Matrigel developed into cystic structures formed by differentiated ductal cells (Gittes et al., 1996). In our study we have used reduced growth factor Matrigel, which contains the same basement membrane components than Matrigel but has been greatly depleted in growth factors. Apparently our culture conditions are less favourable to ductal differentiation. However, a few ductal cells were detected in the explants treated simultaneously with FGF10 and compound 1 suggesting that somehow, the blockage of Notch signalling could be required to allow ductal differentiation. It must be noted also that, as in the FGF10-treated explants, differentiated ductal cells were not detected in the $p P d \times 1-F G F 10$ transgenic mice.

The role of the Notch pathway in the control of pancreatic differentiation is now well established. Loss of function of various Notch pathway genes (Hes1, Delta1, RBPjk) leads to premature and massive differentiation of the pancreatic progenitors into endocrine cells (Apelqvist etal., 1999;Gradwohl etal., 2000;Jensen et al., 2000). A similar phenotype was observed in transgenic mice expressing the bHLHL trascription factor $\mathrm{Ngn} 3$ (a gene usually repressed by Notch activation) under the control of the Pdx1 promoter ( $p P d x 1-N g n 3$ ), (Apelqvist et al., 1999). Moreover, when a Notch-IC transgene is activated in the developing mouse pancreas using the $\mathrm{Pdx} 1$ promoter, both endocrine and exocrine differentiation are repressed, suggesting that Notch has an inhibitory role in the control of the differentiation of both lineages (Murtaugh et al., 2003). It has been suggested that maintenance of the pancreatic progenitors in an undifferentiated state in the pPd $\times 1$-FGF10 mice could result of an eventual effect of FGF10 in inducing persistent activation of the Notch pathway. Our study corroborates this hypothesis. Hes1, a target gene of the Notch pathway, was rapidly downregulated in the isolated $\mathrm{E} 10.5$ pancreatic epithelia, but its expression persisted in the explants treated with FGF10. Moreover, the $\gamma$-secretase inhibitor, compound 1 , downregulated Hes 1 expression and considerably reduced the growth and the number of undifferentiated cells in the FGF10-treated pancreatic epithelia. Thus, the inhibition of the Notch pathway prevents the effect of FGF10 on the proliferation and the maintenance of the pancreatic progenitors in an undifferentiated state. Therefore, the Notch pathway is required as a downstream mediator of the FGF10 signalling in pancreatic precursors. We do not know how FGF10 maintains the Notch activation. It has been suggested, based on the expression of the Notch ligand genes Jagged 1 and Jagged 2 in the undifferentiated pancreatic epithelium of the $p P d \times 1-F G F 10$ mice, that FGF10 could induce the expression of these ligands (Norgaard et al., 2003). FGF10 could also down-regulate repressors of Notch activity like Sel1 (Hart et al., 2003) or up regulate Notch expression. In this regard, it has been shown that the FGF1 and FGF2 induce the proliferation and inhibit the differentiation of neuroepithelial precursors trough Notch signalling. Both FGFs efficiently up-regulate the expression of Notch 1 in these neuronal precursors (Faux et al., 2001). However, our RT-PCR analysis did not show any major differences in the levels of expression of these genes between the FGF10-treated and untreated pancreatic epithelia.

It is noteworthy that untreated isolated pancreatic epithelia showed in vitroan outcome similar to that of the pancreas of mice deficient for different genes of the Notch pathway, or the $p P d \times 1$ Ngn3 mice. That is, arrested growth, accelerated and almost total differentiation of the pancreatic epithelium into endocrine cells and also, as we observed in our study, rapid down-regulation of Hes1. This suggests that the mesenchyme not only provides signals necessary for the growth of the pancreatic epithelium but it can also regulate the maintenance of the pancreatic progenitors in an undifferentiated state via the Notch pathway. In the developing pancreas FGF10 is essentially produced by the pancreatic mesenchyme, while its receptor FGFR2b is expressed only in the epithelial cells (Miralles et al., 1999). Thus, the pattern of expression of FGF10 is consistent with the hypothesis that FGF10 could be the mesenchymal factor responsible of the maintenance of the Notch signalling. Studies on the development of other organs have also implicated the FGFs in the self-renewal of progenitor cells via the Notch pathway. Of particular interest, in this context, are several studies showing that FGF10 is capable of preventing the differentiation of the odontoblasts by inducing Notch signalling (Mitsiadis et al., 1997;Mustonen et al., 2002). These studies
A
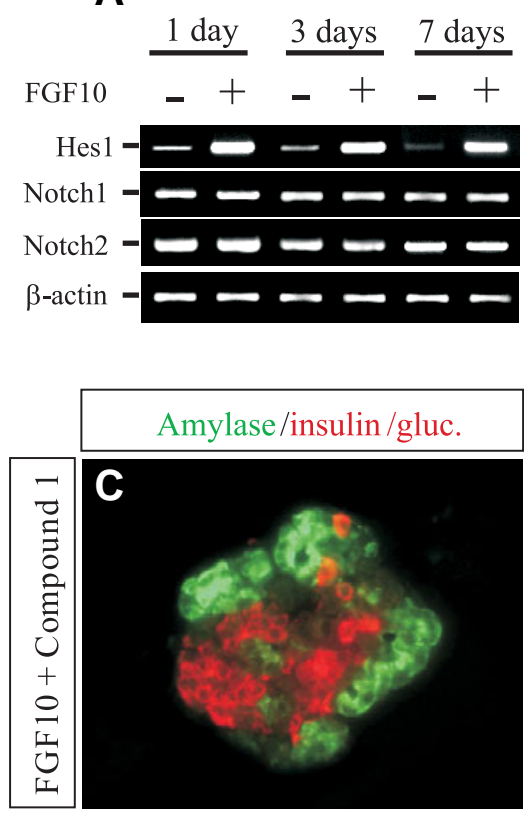

B

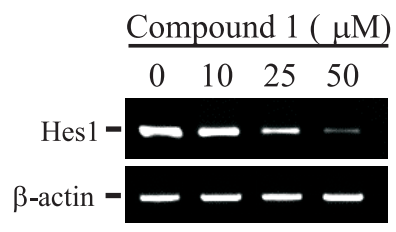

Fig. 7. Requirement for Notch signaling in FGF10 mediated expansion and maintenance of undifferentiated pancreatic progenitors. (A) Semiquantiative RT-PCR analysis demonstrating Hes 1 induction by Notch activation in the pancreatic epithelium cultured with FGF10. (B) RT-PCR demonstrating that the $\gamma$ secretase inhibitor compound 1 efficiently down-regulates Hes 1 in FGF10-treated explants. (C,D) Immunohistological analysis of the development of FGF10-treated pancreatic epithelia cultured in the presence of compound 1. (C) Double immunofluorescence staining for amylase (green) and insulin+glucagon (red). (D) Staining for DBA lectin (green) and insulin+glucagon (red). In these explants the inactivation of the Notch pathway resulted in arrested growth and massive differentiation of the pancreatic progenitors. 
A

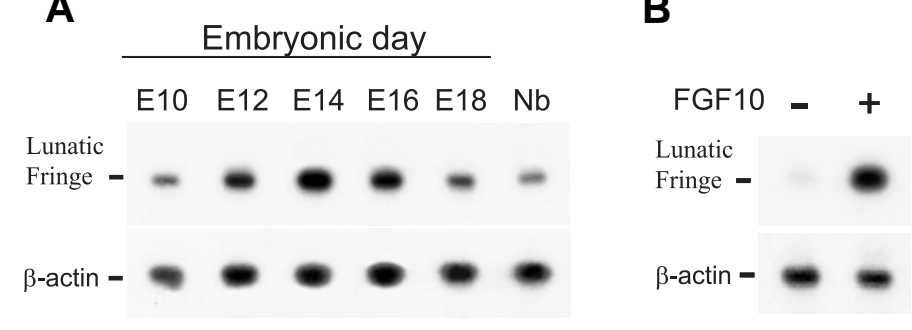

Fig. 8. FGF10 induces Lunatic fringe expression in the isolated pancreatic epithelium. (A) Semiquantitative RT-PCR analysis showing Lunatic fringe expression throughout pancreatic development. (B) RT-PCR demostrating that Lunatic fringe expression is downregulated in the cultured E10.5 isolated pancreatic epithelium and induced in the pancreatic explants treated with FGF10. Nb, newborn pancreas.

showed that Hes 1 expression in the dental precursors is dependent on mesenchymal signals and that FGF10 induces Hes1 expression in explants of isolated dental epithelium. In these explants FGF10 also induces the expression of Lunatic fringe, an enhancer of Notch activity. Interestingly, we have found that Lunatic fringe is expressed throughout pancreatic development and that its maximal expression occurs between E12 and E16, a period corresponding to the expansion of the population of pancreatic precursors. This period coincides with the maximal expression of FGF10 by the pancreatic mesenchyme. Moreover, FGF10 induces Lunatic fringe expression in the E10.5 isolated dorsal pancreatic epithelium. Thus, in vivo FGF10 could maintain Notch activity in the pancreatic precursors by inducing Lunatic fringe.

The present and previous studies indicate that FGF10 is able to maintain active Notch signalling. However, other signalling pathways are also probably implicated in Notch control. In this respect, a recent study has shown that TGF $\alpha$ can induce Notch activation in explant cultures of pancreatic acinar cells. Upon treatment with TGF $\alpha$ these cells expressed high levels of Pdx1 and Hes 1 and underwent acinar to ductal metaplasia (Miyamoto et al., 2003). The study of the interactions between the Notch pathway and other signalling cascades implicated in pancreas development will be crucial to further unravel the mechanisms controlling the self-renewal of the pancreatic precursors.

\section{Materials and Methods}

\section{Animals}

Pregnant mice were obtained from our own breeding facilities (Institut Cochin, France). The morning of the discovery of the vaginal plug was designed as E0.5. Pregnant mice at E10.5 were killed by cervical dislocation. The embryos were harvested and the dorsal pancreatic rudiments were dissected as previously described (Gittes and Galante, 1993).

\section{Explants culture of embryonic pancreas}

To remove the mesenchyme, the pancreatic rudiments were incubated for $10 \mathrm{~min}$ at room temperature in $0.6 \mathrm{U} / \mathrm{ml}$ of dispase II (Roche). After this treatment the epithelium was carefully separated from the surrounding mesenchyme using tungsten needles. Three or four pancreatic epithelia were embedded in $20 \mu$ l of growth factor reduced Matrigel $\mathrm{I}^{\mathrm{TM}}$ or rat-tail type I Collagen gel (both from BD Biosciences). Matrigel was used diluted 1:1 in culture medium. After polymerization the gels were covered with $200 \mu \mathrm{l}$ of culture medium. The culture medium was: DMEM:F12 supplemented with glutamine, $1 \% \mathrm{FCS}$, penicillin:streptomycin and $10 \mathrm{mg} / \mathrm{ml}$ serum albumin.

Human recombinant FGF-10 (R\&D Systems) was diluted in PBS containing $0.1 \%$ serum albumin. The growth factor, was added to the culture medium at a final concentration of $100 \mathrm{ng} / \mathrm{ml}$. The medium was changed every day and fresh FGF10 added. In some experiments 10 $\mu \mathrm{mol}$ BrdU (5-Bromo-deoxy-Uridine) was added to the culture medium for $3 \mathrm{~h}$ to label the explants and allow the study of cell proliferation.

\section{Immunohistochemistry}

Immunohistochemistry was performed on frozen sections ( $5 \mu \mathrm{m}$ thick). On the day of staining, the slides were dried at $37^{\circ} \mathrm{C}$ for $30 \mathrm{~min}$. Sections were permeabilized for 30 min in $0.1 \%$ Triton X-100 in PBS and blocking of unspecific reactivity was performed for $1 \mathrm{~h}$ in $3 \%$ BSA in PBS. Primary antibodies where added at the appropriate dilutions in $0.1 \%$ Triton X-100/ $3 \%$ BSA in PBS and left to react overnight. The next morning the excess of antibody was removed and the sections were washed for $3 \times 5 \mathrm{~min}$ in $0.1 \%$ Tween 20-PBS. Appropriate fluorescent secondary antibodies were then added and incubated for $1 \mathrm{~h}$. After extensive washing in $0.1 \%$ Tween 20-PBS ( 5 washes $\times 5 \mathrm{~min}$ ), the sections were washed for $5 \mathrm{~min}$ in PBS and mounted with VectashieldTM (Vector). The sections were examined and photographed using a fluorescence microscope (Eclipse E800, Nikon) equipped with a digital camera (DXM1200, Nikon) and adapted image acquisition software (ACT-1, Nikon). The Antibodies used in this study, source and dilution were as follows: rabbit anti-Pdx1, C. Wright, USA, 1:1000; rabbit anti-Nkx6.1, M. German, USA, 1:500; rabbit anti-p48/ Ptf1 $\alpha, H$. Edlund, Sweden, 1:1000; mouse anti-Somatostatin, Dako, 1:500; mouse anti-Glucagon, Sigma, 1:2000; mouse anti-Insulin, Sigma, 1:1000; guinea-pig anti-insulin, Dako, 1:2000; rabbit anti-Pancreatic peptide, Dako, 1:1200; rabbit anti-Amylase, Sigma, 1:2000; rabbit antiTrypsin, C. Figarella, France, 1:000: rabbit anti-carboxypeptidase A, Anawa, 1:1000; mouse anti- $\beta$-catenin, BD Biosciences, 1:200; mouse anti-BrdU, Sigma, 1:200.

The fluorescent secondary antibodies were: fluorescein anti-guinea pig antibodies (Dako), 1:500; and the following from Jackson ImmunoResearch: fluorescein anti-rabbit antibodies, 1:500; fluorescein anti-mouse antibodies, 1:200; Texas-red anti-rabbit antibodies, 1:500; Texas-red anti-mouse antibodies, 1:200; Texas-red anti-guineapig antibodies, 1:500.

Fluorescein-labelled DBA lectin was purchased from Sigma, 1:100.

\section{In situ hybridization}

For ISH, explants and embryonic or foetal mouse pancreas were fixed in $4 \%$ paraformaldehyde and embedded in OCT. Frozen sections were hybridized with digoxygenin-labeled riboprobes. The hybridized probes were detected using AP-conjugated anti-digoxigenin antibodies (Roche). Signal was visualized by using the NBT/BCIP substrate (Roche). Probes against Hes1 and Ngn3 were a generous gift of Dr G. Gradwohl (INSERM, France). The $p 48$ probe was kindly provided by Dr. P.K. Wellauer (Institute for Experimental Cancer Research, Lausanne).

\section{Quantitative analysis}

To determine the relative proportions of the different cell types in the rudiments we measured the surface area staining positively for each specific cell marker and divided by the total area of the rudiment section. The analysis of cell surface areas was done on digitalized images of pancreatic rudiments using NIH image software. The data shown represent the mean values (in \%) of the relative surface area occupied by each cell type in different sections of the rudiments analyzed. The total proliferation index in the rudiments was calculated as the number of $\mathrm{BrdU}+$ nuclei/area. For the analysis of the proliferation of each cell type the rudiments were co-stained with anti-BrdU and anti-insulin, antiglucagon or anti-amylase antibodies. The cells were counted and the percentage of each cell type co-staining positively with BrdU calculated. 
Results are presented as the mean \pm SEM. Statistical differences were determined using a Student's t test for independent samples.

\section{Reverse transcriptase-PCR analyses}

Pancreatic rudiments were dissolved in Trizol (Invitrogen) and total RNA was extracted according to the manufacturers instructions. Reverse transcriptase reactions were done using the Cloned AMV/First-strand Synthesis Kit (Invitrogen). The oligonucleotides used for amplification were:

Amylase (forward) 5'-CATTGTTGCACCTTGTCACC-3'

(reverse) 5'-TTCTGCTGCTTTCCCTCATT-3'

Carboxypeptidase A (forward) 5'-GCAAATGTGTGTTTGATGC-3'

(reverse) 5'-ATGACCAAACTCTTGGACCG-3'

Carbonic ahydrase // (forward) 5'-TTGATGACTCTCAGGACAATG-3'

(reverse) 5'-CTTGTGAGGCAGGTCCAATCTTC-3'

CFTR (forward) 5'AAGTATTGGACAACTTGTTAGTC-3'

(reverse) 5'-TAATTCCCCAAATCCCTCCTC-3'

Pdx1(forward) 5'-TCGCTGGGATCACTGGAGCA-3'

(reverse) 5'-GGTTCCGCTGTGTAAGCACC-3'

p48/Ptfla (forward) 5'-AGGAAAGGGAGTGCCCTGCAAG-3'

(reverse) 5'-GGCCCAGAAGGTCATCATCTGC-3'

Neurogenin-3 (forward) 5'-TGGCGCCTCATCCCTTGGATG-3'

(reverse) 5'-CAGTCACCCACTTCTGCTTCG-3'

nsulin (forward) 5'-TAGTGACCAGCTATAATCAGAG-3'

(reverse) 5'-ACGCCAGGTCTGAAGGTCC-3'

Notch1 (forward) 5'-CTGGTTCCCTGAGGGTTTCAA-3'

(reverse) 5'-GGAACTTCTTGGTCTCCAGGT-3'

Notch2 (forward) 5'-CAACATGGGCCGCTGTCCTC-3'

(reverse) 5'-CACATCTGCTTGGCAGTTGATC-3'

Jagged1 (forward) 5'-TGTGTGAAGTTGGAAGCATCC-3'

(reverse) 5'-ACCTTGAGCTTGGTAATAGCA-3'

Jagged 2 (forward) 5'-AAGGACATACTCTACCAGTGC-3'

(reverse) 5'-ACGTCCTTGGTACTTCTGACG-3'

Hes 1 (forward) 5'-TCAACACGACACCGGACAAACC-3'

(reverse) 5'-GGTACTTCCCCAACACGCTCG-3'

Lunatic fringe (forward) 5'-GTGCATAGCCTCTCCGAGTACTTCA-3'

(reverse) 5'-CTCCTCCGGTGGCAAACCAAAAGTG-3'

Typically 30 cycles of amplification were performed. Amplification parameters were: 1 min denaturation step at $94^{\circ} \mathrm{C}, 1 \mathrm{~min}$ annealing step at $57^{\circ} \mathrm{C}$ and $1 \mathrm{~min}$ elongation step at $72^{\circ} \mathrm{C}$. The products of amplification were run in $2 \%$ agarose gels and either photographed or subjected to Southern-blot analysis.

\section{Notch pathway inhibition}

Notch pathway inhibition was achieved using a peptidomimetic inhibitor of $\gamma$-secretases, compound 1 (Sigma). FGF10-treated rudiments were simultaneously treated with compound 1 at 10,25 or $50 \mu \mathrm{M}$. Control explants were treated with DMSO vehicle.

\section{Acknowledgments}

We are indebt to Dr. F. Langa for manuscript-reading and helpful discussions. We thank Drs. C. Figarella, H. Edlund, G. Gradwohl, M. German, P.K. Wellauer and C. Wright for providing valuable materials. This research was supported by INSERM and AFM funds.

\section{References}

APELQVIST, A., LI, H., SOMMER, L., BEATUS, P. ANDERSON, D.J., HONJO, T., HRABE DE ANGELIS, M., LENDAHL, U. and EDLUND, H. (1999). Notch signalling controls pancreatic cell differentiation. Nature 400: 877-81.

BEATUS, P. and LENDAHL, U. (1998). Notch and neurogenesis. J Neurosci Res 54: $125-36$

BHUSHAN, A., ITOH, N., KATO, S., THIERY, J.P., CZERNICHOW, P., BELLUSCI, S. and SCHARFMANN, R. (2001). Fgf10 is essential for maintaining the proliferative capacity of epithelial progenitor cells during early pancreatic organogenesis. Development 128: 5109-17.

CELLI, G., LAROCHELLE, W.J., MACKEM, S., SHARP, R. and MERLINO, G. (1998). Soluble dominant-negative receptor uncovers essential roles for fibroblast growth factors in multi-organ induction and patterning. EMBO J17: 164255.

DE STROOPER, B., ANNAERT, W., CUPERS, P., SAFTIG, P., CRAESSAERTS, K., MUMM, J.S., SCHROETER, E.H., SCHRIJVERS, V., WOLFE, M.S., RAY, W.J. et al. (1999). A presenilin-1-dependent gamma-secretase-like protease mediates release of notch intracellular domain. Nature 398: 518-22.

EDLUND, H. (2002). Pancreatic organogenesis-developmental mechanisms and implications for therapy. Nat Rev Genet 3: 524-32.

ESNI, F., GHOSH, B., BIANKIN, A.V., LIN, J.W., ALBERT, M.A., YU, X., MACDONALD, R.J., CIVIN, C.I., REAL, F.X., PACK, M.A. et al. (2004). Notch inhibits ptf1 function and acinar cell differentiation in developing mouse and zebrafish pancreas. Development 131: 4213-24.

FAUX, C.H., TURNLEY, A.M., EPA, R., CAPPAI, R. and BARTLETT, P.F. (2001). Interactions between fibroblast growth factors and notch regulate neuronal differentiation. J Neurosci21: 5587-96.

FISHER, A. and CAUDY, M. (1998). The function of hairy-related bhlh repressor proteins in cell fate decisions. Bioessays 20: 298-306.

GITTES, G. and GALANTE, P. (1993). A culture system for the study of pancreatic organogenesis. J Tiss Cult Meth 15: 23-28.

GITTES, G., GALANTE, P., HANAHAN, D., RUTTER, W. and DEBAS, H. (1996). Lineage specific morphogenesis in the developing pancreas: Role of mesenchymal factors. Development 122: 439-447.

GITTES, G. and RUTTER, W. (1992). Onset of cell-specific gene expression in the developing mouse pancreas. Proc Natl acad Sci USA 89: 1128-1132.

GOLOSOW, N. and GROBSTEIN, C. (1962). Epitheliomesenchymal interaction in pancreatic morphogenesis. Dev Bio/4: 242-255.

GRADWOHL, G., DIERICH, A., LEMEUR, M. and GUILLEMOT, F. (2000). Neurogenin 3 is required for the development of the four endocrine cell lineages of the pancreas. Proc Nat/ Acad Sci U S A 97: 1607-11.

HALD, J., HJORTH, J.P., GERMAN, M.S., MADSEN, O.D., SERUP, P. and JENSEN, J. (2003). Activated notch1 prevents differentiation of pancreatic acinar cells and attenuate endocrine development. Dev Bio/260: 426-37.

HART, A., PAPADOPOULOU, S. and EDLUND, H. (2003). Fgf10 maintains notch activation, stimulates proliferation and blocks differentiation of pancreatic epithelial cells. Dev Dyn 228: 185-93.

HART, A.W., BAEZA, N., APELQVIST, A. and EDLUND, H. (2000). Attenuation of fgf signalling in mouse beta-cells leads to diabetes. Nature 408: 864-8.

JENSEN, J. (2004). Gene regulatory factors in pancreatic development. Dev Dyn 229: $176-200$

JENSEN, J., PEDERSEN, E.E., GALANTE, P., HALD, J., HELLER, R.S., ISHIBASHI, M., KAGEYAMA, R., GUILLEMOT, F., SERUP, P. and MADSEN, O.D. (2000). Control of endodermal endocrine development by hes-1. Nat Genet24: 36-44.

KIM, S.K. and MACDONALD, R.J. (2002). Signaling and transcriptional control of pancreatic organogenesis. Curr Opin Genet Dev 12: 540-7.

KURODA, K., TANI, S., TAMURA, K., MINOGUCHI, S., KUROOKA, H. and HONJO, T. (1999). Delta-induced notch signaling mediated by rbp-j inhibits myod expression and myogenesis. J Biol Chem 274: 7238-44.

LEE, J.C., SMITH, S.B., WATADA, H., LIN, J., SCHEEL, D., WANG, J., MIRMIRA, R.G. and GERMAN, M.S. (2001). Regulation of the pancreatic pro-endocrine gene neurogenin3. Diabetes 50: 928-36.

MIRALLES, F., CZERNICHOW, P., OZAKI, K., ITOH, N. and SCHARFMANN, R. (1999). Signaling through fibroblast growth factor receptor $2 b$ plays a key role in the development of the exocrine pancreas. ProcNat/Acad SciUS A96: 626772.

MIRALLES, F., CZERNICHOW, P. and SCHARFMANN, R. (1998). Follistatin regulates the relative proportions of endocrine versus exocrine tissue during pancreatic development. Development 125: 1017-24.

MITSIADIS, T.A., HENRIQUE, D., THESLEFF, I. and LENDAHL, U. (1997). Mouse serrate-1 (jagged-1): Expression in the developing tooth is regulated by epithelial-mesenchymal interactions and fibroblast growth factor-4. Development 124: $1473-83$. 


\section{F. Miralles et al.}

MIYAMOTO, Y., MAITRA, A., GHOSH, B., ZECHNER, U., ARGANI, P., IACOBUZIODONAHUE, C.A., SRIURANPONG, V., ISO, T., MESZOELY, I.M., WOLFE, M.S. et al. (2003). Notch mediates tgf alpha-induced changes in epithelial differentiation during pancreatic tumorigenesis. Cancer Ce//3: 565-76.

MURTAUGH, L.C., STANGER, B.Z., KWAN, K.M. and MELTON, D.A. (2003). Notch signaling controls multiple steps of pancreatic differentiation. Proc Nat/ Acad SciUS A 100: 14920-5.

MUSTONEN, T., TUMMERS, M., MIKAMI, T., ITOH, N., ZHANG, N., GRIDLEY, T. and THESLEFF, I. (2002). Lunatic fringe, fgf and bmp regulate the notch pathway during epithelial morphogenesis of teeth. Dev Bio/248: 281-93.

NORGAARD, G.A., JENSEN, J.N. and JENSEN, J. (2003). Fgf10 signaling maintains the pancreatic progenitor cell state revealing a novel role of notch in organ development. Dev Bio/264: 323-38.

POWERS, C.J., MCLESKEY, S.W. and WELLSTEIN, A. (2000). Fibroblast growth factors, their receptors and signaling. Endocr Relat Cancer 7: 165-97.

PULKKINEN, M.A., SPENCER-DENE, B., DICKSON, C. and OTONKOSKI, T. (2003). The iiib isoform of fibroblast growth factor receptor 2 is required for proper growth and branching of pancreatic ductal epithelium but not for differentiation of exocrine or endocrine cells. Mech Dev120: 167-75.
REVEST, J.M., SPENCER-DENE, B., KERR, K., DE MOERLOOZE, L., ROSEWELL, I. and DICKSON, C. (2001). Fibroblast growth factor receptor 2-iiib acts upstream of shh and fgf 4 and is required for limb bud maintenance but not for the induction of fgf8, fgf10, msx1, or bmp4. Dev Bio/231: 47-62.

RUTTER, W., PICTET, R., HARDING, J., CHIRGWIN, J., MACDONALD, R. and PRZYBYLA, A. (1978). An analysis of pancreattic development: Role of mesenchymal factor and other extracellular factors. Symp Soc Dev Bio/35: 205-227.

WESSELS, N. and COHEN, J. (1967). Early pancreas organogenesis: Morphogenesis, tissue interactions and mass effects. Dev Bio/15: 237-270.

WOLFE, M.S., XIA, W., MOORE, C.L., LEATHERWOOD, D.D., OSTASZEWSKI, B., RAHMATI, T., DONKOR, I.O. and SELKOE, D.J. (1999). Peptidomimetic probes and molecular modeling suggest that alzheimer's gamma-secretase is an intramembrane-cleaving aspartyl protease. Biochemistry 38: 4720-7.

Received: August 2005

Reviewed by Referees: September 2005

Modified by Authors and Accepted for Publication: October 2005 